\title{
Organizational Activities Through Social Media Communication With Direct Communication
}

\author{
Derius Neuga $^{1}$, Larentiu Nicoulae Silviu ${ }^{1}$ \\ Email: nicolevie.silvie12@sas.unibuc.ro
}

${ }^{1}$ Faculty of Sociology and Social Work, University of Bucharest, Romania

\begin{abstract}
Interpersonal contact or contact that is inextricably linked to mass media. When individuals upload things and communicate with other participants, internal contact happens simultaneously with public communication, since whatever is shared can be automatically appreciated and seen by a large audience. With the potential for private information to become available, it is important to pay more attention to the role of social media. Not to stifle its development, but to optimize its utility. It is critical to improve each user's self-awareness, since what they upload will influence their self-image and the relationships they have with other parties. The freedom of expression and the freedom to communicate that social media provides should be used prudently by its consumers. When we share or disseminate knowledge, we must exercise caution and introspection. In other words, while contact through social networking has expanded and become more versatile, this versatility must be reined in to truly enjoy the benefits. Naturally, if we understand the components of organizational communication, as we communicate inside the organization, we can understand our status in light of the scenario and circumstances. The advancement of the internet facilitated the emergence of modern modes of communication within organizations. Social networking has arrived and is reshaping the way we communicate in today's culture. There is no restriction to the correspondence of distance, time, and space.
\end{abstract}

Keywords: Interpersonal communication, Social Media \& Organizations

Received: March 3, 2021

Revised: March 28, 2021

Accepted: April 20, 2021

\section{Introduction}

It is almost difficult to avoid public attention in today's modern world. If we are aware of it or not, media and its material are an integral part of human existence. The media's influence has been more complex and developed over time. Initially, information contact was one-way, in the context that media enthusiasts might only appreciate the news provided by media outlets. However, as time passes, average citizens as information connoisseurs can no longer simply appreciate the quality of the media to which they are subjected, but can still contribute to the media's content development.

The growth and growth of the internet has resulted in the proliferation and development of new modes of communication in culture. Social networking has arrived and is reshaping the way we communicate in today's culture. There is no restriction to the correspondence of distance, time, and space. It may occur anywhere, at any moment, without the need for a face-to-face meeting. And social networking may obliterate social class, which is often a stumbling block to contact. With the use of social media platforms such as Twitter, Facebook, and Google+, individuals may communicate without physically meeting.

Communication is no longer hampered by distance. The time difference between our last meetings was no longer a problem. Friends that haven't seen each other in over a decade will reconnect and re-establish contact. Additionally, due to social media's ease of usage, nearly

Copyright ( 2021 , International Journal Papier Public Review, Under the license CC BY-SA 4.0 DOI: https://doi.org/10.47667/ijppr.v2i2.84 
everyone can reach and utilize it. The landscape has shifted dramatically as a result of social media. Pervert a great deal of owned ideas and theories. The degree or level of contact has been consolidated into a single container dubbed social networking / social media. Consequences must also be considered, as social media is gradually allowing individuals to publicly share their views. However, self-control must be possessed in order for the right they possess to not break limits or insult third parties.

\section{Communication Components}

To conceptualize communication in an organisation, it is necessary to first understand the communication aspect. In general, an Effective Communication Process consists of five major components. Both elements include the following: (1) communicators or message senders; and (2) communicants or message receivers. (3) Data or messages; (4) The medium or channel of communication; and (5) Reactions to data or messages. The five components are often present in intra-organizational cooperation, although the individuals that comprise each of these components vary according to the case and circumstances. In Organizational Meetings, for instance.

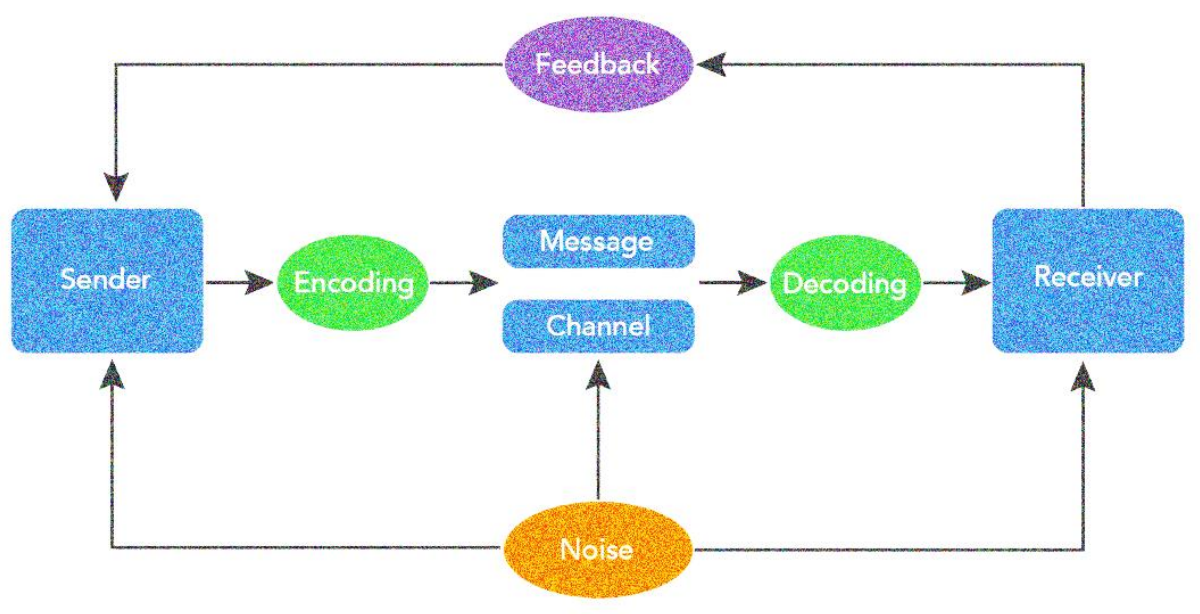

Figure 1. Communication Components Model

Source: Organizational Behavior / Human Relations (Lumencandela, nd)

Communicators are administrators or representatives of organizations; communicants are individuals deemed worthy of offering assistance; and information given relates to assistance demands (Gratz \& Salem, 1981; Gangel \& Canine, 2002). The media may take the form of television, radio, print, electronic, or online media, as well as electronic devices. Feedback is the response provided by goals, in this case those perceived to be offering assistance. At this early stage of the concept, someone filling in the part must possess the ability to become a successful filler. If you're going to be a communicator, make sure you're a successful communicator. If you're going to be a communicant, make sure you're a decent communicator as well.

\section{Characteristics of the Organization}

Naturally, if we understand the components of organizational communication, as we communicate inside the organisation, we can understand our status in light of the scenario and circumstances. After identifying the coordination elements and their locations, the next step is to investigate the organization's characteristics. The organization's features are as follows: Available, the organization's character must be transparent to everyone, welcome everyone, and ensure that all content is accessible to all members. Dynamic, the organisation must be 
capable of adapting to and incorporating all developments that exist both internally and externally. Awareness, the individuals who work in the company must be knowledgeable regarding the organization's requirements. Purpose, an organisation's formation must have a purpose, such as being a political organization or a non-governmental organization. Structurewise, the organisation would provide administration in place to oversee all operating operations. Typically, the corporate management system corresponds to the organization's scale. However, they work similarly to the general leader, secretary, item, and representatives. Integrity, Ethics and politeness are critical components of every organisation. As a result, all correspondence must be conducted in an appropriate, accurate, legal, and respectful manner.

\section{Barriers to Organizational Communication}

Understanding the Barriers to Organizational Communication is the first phase of How to Communicate Effectively, and they are well conceptualized. Several communication barriers that must be understood and recognized in organisations include the following: Technical Obstacles, Media organisations typically include certain technological equipment, which includes technologies used to transmit content, communication equipment, communication protocols or regulations, information provider capabilities in conveying communications, and organizational representatives' participation.

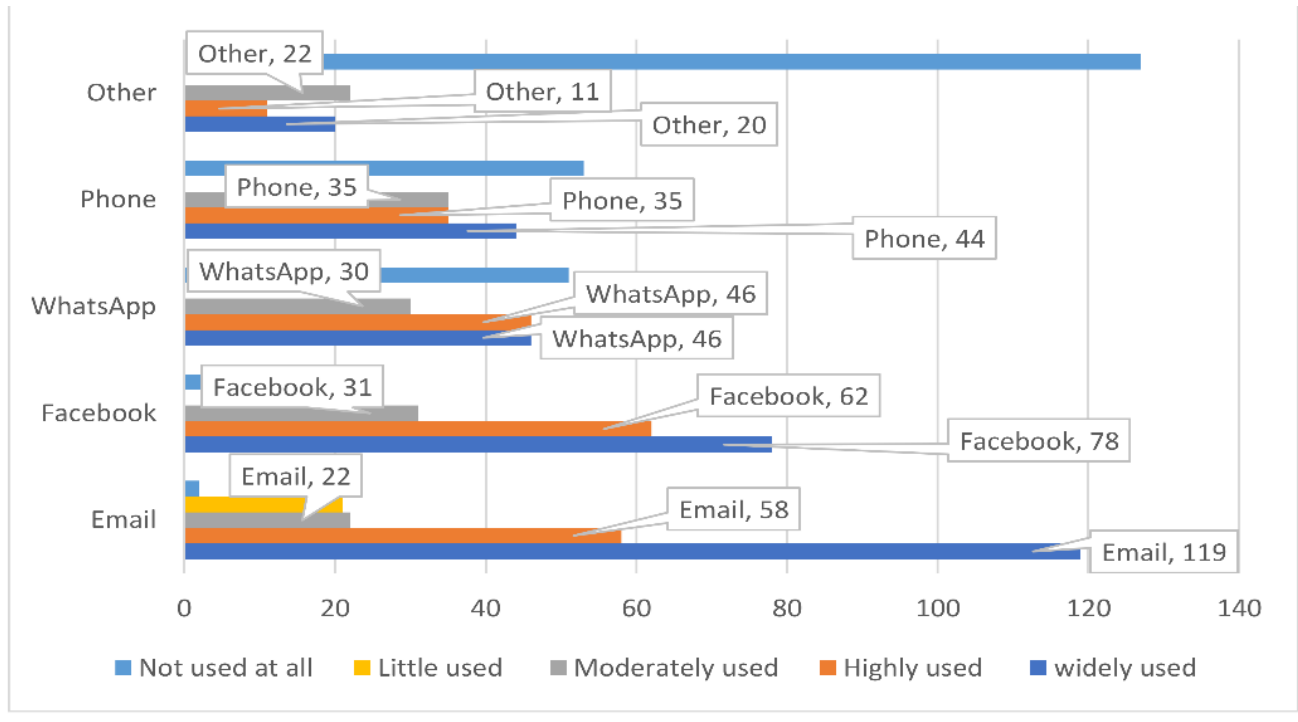

Figure 2. Online Communication system used in Romania

Source: Edelhauser \& Lupu-Dima (2020)

Semantic Obstacles, Semantic barriers are impediments that arise as a result of a discrepancy between the source and recipient of knowledge, often known as a confusion. Therefore, when conceptualizing correspondence inside an organisation, make an effort to utilize simple terms or sentences. Logical Obstacles, Logical impediments to understanding the facts conveyed in the context of feelings, prejudices, emotions, and expectations. To overcome this barrier, the communicator's capacity to explain knowledge before it is fully understood by the communicant is needed.

\section{Social Media Communication in Organizations}

Organizational collaboration using digital media. According to the wise, everything happens in this universe except for change itself. Similarly, corporate correspondence is stagnant and is conducted in a vacuum (growing in a vacuum). Organizational collaboration is entering a modern age that is characterized by a radical shift in communication processes. It is referred to 
as the Digital Age or the Post-Industrial Age. This exponential growth in new technologies has resulted in a transition on a par with the Industrial Revolution of the 1800s. Since 1994, when the internet and Web 2.0 became widely used by the public, corporate organisations have begun to apply information and communication technology (ICT) to internal communication. Previously, contact inside the organization was more reliant on the back office and the front office; today, it is more reliant on a virtual office.

SOCIAL NETWORKS

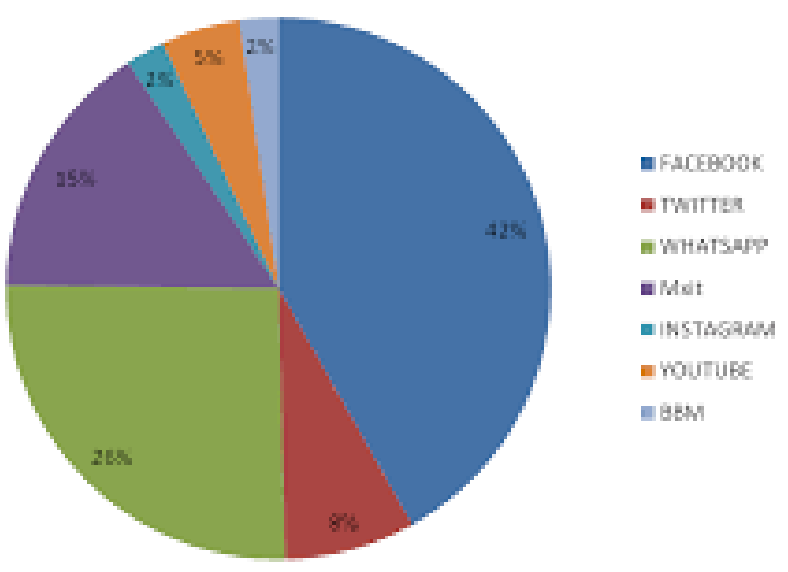

Figure 3. Youth social media communication preferences

Source: Vuyisile Sikelela Hlatshwayo (2014)

\section{Communication Proficiency in Social Media}

Intrapersonal contact, oral communication, community communication, public communication, and mass communication both fall into the category of communication. Each degree of contact is distinct in its own way. The growth of digital media has resulted in shifts and developments in mass communication theory (Bandura, A. (2001; Weingart et al., 2000). The previously recognized features of media have been incorporated into the digital media. This is as a result of the development of mass-self communication. In digital technology, interpersonal conversation is combined with mainstream communication (Konijn et al., 2008). Since it enters a worldwide audience, it is considered mass communication; however, since actual communications are produced, guided, and ingested individually, it is considered interpersonal communication.

Interpersonal contact is described as communication between two individuals or as communication that is contextually interpersonal. And if it exists in small communities, contextual interpersonal contact is very distinct from community communication. Interpersonal dialogue necessitates the active participation of both participants. Interpersonal contact is effectively terminated as one side withdraws from the dialogue. Obviously, this extends to social networking as well. In social networking, contact is not collaborative if the group inviting the other party to talk withdraws from the current discussion. Without knowledge sharing, the information source, in this case the user of the social network account, speaks only in one direction; but, if another individual reacts to what he posts and an encounter happens, interpersonal contact occurs.

Online networking facilitates the formation of intimate interpersonal connections for many individuals. Since online networking encourages individuals to be more courageous in voicing their opinions and allowing themselves to be understood by others. Social network communication is not time-bound; it can take place at any time of day or night, and all parties concerned can participate actively. Additionally, the parties concerned are not limited by space; 
they may interact with everyone on the planet. This is not achievable in face-to-face conversation, even even by utilizing more traditional modes of communication such as the telephone, owing to expense and time disparities.

Online networking is perceived to be more cost effective, quicker, and smoother in this respect. According to Simpson, (2015); West et al. (2010); Liu et al. (2021). Mass communication is the most extensive form of communication, covering the greatest number of coordination goals (Aggarwal \& Gupta, 2001). Mass media is the dissemination of messages to a broad and diverse potential population through the use of newspapers, magazines, television, radio, and the internet. This is indeed a condition that exists in social media.

What an individual discloses on social media can be used by a large number of people, resulting in mass communication. Interpersonal correspondence and mass communication was merged with social networking. When one party uploads something, the other party reacts, which results in an encounter and verbal contact. Simultaneously, anytime someone uploads something, it may be seen and enjoyed widely, resulting in mass communication, which does not entail the active participation of both stakeholders.

\section{Intra-Organizational Coordination}

According to Kim, (2001) Direct contact effectiveness is determined by five general characteristics, including transparency (openness), empathy, supportiveness, a constructive mindset (positiveness), and equality.

Transparency (Opennes) Openness is a characteristic that encompasses at least three facets of direct contact. To begin, good interpersonal communicators must be receptive to those with whom they communicate. This does not suggest that any of his biographies can be automatically opened. Although this might be amusing, it seldom aids in conversation. Empathy (Empathy) is the capacity of an individual to understand what another person is doing at a particular time, through the other person's perspective, or from the other person's perspective.

Sustaining. A supportive partnership is an important direct relationship. A definition focused on the work of Jack Gibb. Positivity (Positive Attitude) In direct contact, we convey a good outlook in at least two ways: (1) by communicating a positive attitude and (2) by actively influencing others with whom we associate. A favorable mood is associated with at least two facets of interpersonal contact.

Equality (Equality) Disparity exists in any case. One individual may be more intelligent than another. Others might be wealthier, more handsome or pretty, or more athletic than you. Nobody is completely fair in everything. Regardless of these disparities, interpersonal contact is more efficient when the environment is balanced.

The primary condition for successful contact is the message's character and personal honesty. Five critical components are required for good communication: the desire to better consider others, the capacity to keep promises, the ability to justify goals, the readiness to apologise honestly for errors, and the ability to demonstrate honesty. The highest level of contact is empathic communication, which means interacting in order to comprehend and comprehend the persona, intentions, and functions of the recipients of the letter.

Communication is critical for corporate progress in terms of both optimizing efficiency and adapting to developments in the current market climate in order for the organisation to succeed and perhaps achieve a competitive edge. The organisation may receive the knowledge it requires by effective contact with entities and parties both within and outside the organization. 
To foster effective collaboration, supervisors and subordinates must take an active position through software and a variety of techniques.

\section{Conclusion}

The virtual world's existence expands the possibilities for each group concerned to live more broadly. Particularly for those who are social media savvy. Many people attempt to demonstrate their presence by constantly updating all current developments through status updates, tweets, notes, and other features in social media. A development that, if it is difficult to convey in the modern world, will become mass consumption in the world of Social Media. Social network communication is becoming more complicated. The two modes of expression converge. The primary condition for successful contact is the message's character and personal honesty. Interpersonal correspondence and mass communication was merged with social networking. When one party uploads something, the other party reacts, resulting in an encounter and interpersonal contact.

\section{References}

Aggarwal, V. B., \& Gupta, V. S. (2001). Handbook of journalism and mass communication. Concept Publishing Company.

Bandura, A. (2001). Social cognitive theory of mass communication. Media psychology, 3(3), 265-299.

Edelhauser, E., \& Lupu-Dima, L. (2020). Is Romania Prepared for eLearning during the COVID-19 Pandemic?. Sustainability, 12(13), 5438.

Gangel, K. O., \& Canine, S. (2002). Communication and conflict management in churches and Christian organizations. Wipf and Stock Publishers.

Gratz, R. D., \& Salem, P. J. (1981). Organizational Communication and Higher Education. AAHE-ERIC/Higher Education Research Report No. 10, 1981.

Kim, H. W. (2001). Modeling inter-and intra-organizational coordination in electronic commerce deployments. Information Technology and Management, 2(3), 335-354.

Konijn, E. A., Utz, S., Tanis, M., \& Barnes, S. B. (Eds.). (2008). Mediated interpersonal communication. Routledge.

Liu, W., Xu, W., \& John, B. (2021). Organizational Disaster Communication Ecology: Examining Interagency Coordination on Social Media During the Onset of the COVID-19 Pandemic. American Behavioral Scientist, 0002764221992823.

Simpson, C. (2015). Science of coercion: Communication research \& psychological warfare, 1945-1960 (Vol. 13). Open Road Media.

Weingart, P., Engels, A., \& Pansegrau, P. (2000). Risks of communication: discourses on climate change in science, politics, and the mass media. Public understanding of science, 9(3), 261-284.

West, R. L., Turner, L. H., \& Zhao, G. (2010). Introducing communication theory: Analysis and application (Vol. 2). New York, NY: McGraw-Hill. 